\title{
SPECTROSCOPIC VARIABILITY IN LATE-TYPE DWARFS USING HIGH S:N SPECTRA
}

\author{
B.H. Foing ${ }^{1}$, J.E. Beckman ${ }^{2}$, G. Bladilo ${ }^{3}$ \\ 1. LPSP/IAS Verrieres, France. \\ 2. Instituto de Astrofisica de Canarias, Tenerife, Spain. \\ 3. Osservatorio Astronomico di Trieste, Trieste, Italy.
}

ABSTRACT. Monitoring of active late-type dwarfs, spectroscopically with high resolution $\left(\lambda / \Delta \lambda \sim 10^{5}\right)$ and high $\mathrm{S}: \mathrm{N}(>300)$ round their activity cycles, principally in the Call $H$ resonance line, offers techniques to explore (a) plage filling factors (b) 3-dimensional chromospheric velocity fields (c) maps of surface activity via "Doppler Imaging". In this paper we deal with the use of spectral signatures to derive plage cover.

\section{INTRODUCTION}

Chromospheric emission flux variability of Call $H$ and $K$ in solar-like stars was studied by O.C. Wilson (1978) in hi s classic work on magnetic activity cycles (periods of years). Short term modulation (periods of days) of this flux was observed by Vaughan et al. (1981) and used by them to compute rotation periods, and hence to investigate the relation period-activity-age. The use of high $S: N$ line profiles instead of fluxes allows us in principle to obtain three new types of information:

(1) The proportion of active (i.e. plage and network) to quiet chromospheric surface cover, given a knowledge of the intrinsic profile emitted by each component.

(2) The presence of velocity fields in three dimensions. The presence of an asymmetry, or the position of a bump in the line profile, and the speeds with which those features change should allow us to distinguish vertical motions from the projected rotational motions of plages.

(3) Combined density and velocity can be combined ("Doppler imaging") to yield maps of chromospheric surfaces, specifying active and quiet regions.

\section{COMPARISON OF $\mathrm{CaH}$ FOR $\alpha$ Cen A AND $\alpha$ Cen $B$}

In Fig. 1 we show an example of the kind of spectroscopic change that can occur due to the change in plage cover during a rotational period. The spectra are of $\alpha$ Cen $B$, taken with the CAT+CES (ESO) at a resolution $\lambda / \Delta \lambda=105$, and with signal to noise at the $H 1$ and $K 1$ minima of 100 and in the continuum of 300 . They span a period of 11 days in June 1985, and 
are in two groups: $8 / 9$ June and $17 / 19$ June, which show little change within each pair. The photospheric spectrum is constant, but the chromospheric emission profile shows a very clear change. The contrast with $\alpha$ Cen $A$ is striking, as shown in Fig. 2, where difference spectra for the two objects are shown. In $\alpha$ Cen $A$ there is no measurable difference to within an impressive $0.3 \%$ RMS error across the spectrum, while in $\alpha$ Cen $B$ there is a "difference profile" which corresponds to a $21 \%$ increment in the $H$ index over minimum phase, and to a FWHM of $33 \mathrm{~km} \mathrm{~s}-1$.
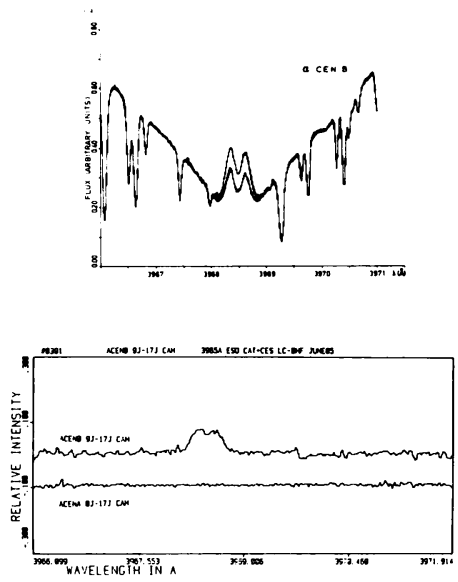

Fig 1: Changes in the $\mathrm{Ca} \mathrm{H}$ emission from the chromosphere of $\alpha$ Cen $B$. Four spectra are superposed. There is no photospheric variation but clear chromospheric changes between $8 / 9$ June and $17 / 19$ June 1985 (2 pairs).

Fig. 2: Differences between spectra of $\alpha$ Cen $B$ and $\alpha$ Cen $A$ from spectra of 9 and 19 June 1985. The clean low-noise subtration for $\alpha$ Cen $A$ gives confidence in the variability data for $\alpha$ Cen $B$.

\section{USE OF PROFILE CHANGES TO SEPARATE ACTIVE FROM QUIET CHROMOSPHERE}

Assuming a chromospheric disc to be composed of three components: a quiet virtually magnetic field-free background, a homogeneously distributed network structure, and magnetically concentrated plages at specific positions we can develop the formalism to distinguish these.

If the resulting line profile $\Phi$ is composed of terms due to the quiet chromosphere with profile $\Phi_{\lambda}^{Q}$, due to the network with profile $\Phi^{N}$, and filling factor $f N$ and due to plages, with profile $\Phi_{\lambda}^{A}$ and filling factor $f P$ the profile will be espressed as

$$
\begin{aligned}
\Phi_{\lambda}(t) & =\Phi_{\lambda}^{Q}\left(1-f^{N}-f^{P}(t)\right)+\Phi_{\lambda}^{A}\left(f^{N}+f^{P}(t)\right) \\
& =\Phi_{\lambda}^{Q}\left(1-f^{N}\right)+\Phi^{A}{ }_{f}^{N}+f^{P}(t)\left(\Phi^{A}-\Phi{ }^{Q}\right)
\end{aligned}
$$

Of course, one cannot use equation (1) directly to find separately all of the unknown parameters. However, careful observations such as those 
exemplified in Fig. 1 for $\alpha$ Cen $B$ show that one can make very sseful approximations. Modulation of the observed profile round a couple of stellar rotation periods shows that $\Phi_{\lambda}(t)$ takes a base-line form, and modulations occur above this; we can assume that this is due to the time variations of the 3rd term on the L.H.S. of (1) as plages appear and disappear via rotation. Terms 1 and 2 remain constant. For truly solar-like stars (G2V) we can then assume a known network filling factor $f \mathrm{~N}$ and known quiescent and active profiles $\Phi^{A}$ and $\Phi^{Q}$ to obtain plage filling factors $f P(t)$. For adjacent spectral types, we have to assume similar values for ${ }^{N}{ }_{\Phi} A$ and $\Phi^{Q}$ and look for self-consistent solutions. These can be cross-checked by using lines other than $\mathrm{Ca} \mathrm{H}$, e.g. $\mathrm{Ca} \mathrm{K}$ or the $\mathrm{Ca}$ IR triplet.

One must remember that maximum variability in the chromospheric emission core is not associated with maximum plage cover and indeed that total plage cover would yield an invariant "high" profile. This contrast effect is shown in the highly active star $70 \mathrm{Oph} \mathrm{A}$, whose $\mathrm{Ca} \mathrm{H}$ profile is shown at high $\mathrm{S}: \mathrm{N}$ in Fig. 3. In this, as in Fig. 1, a logarithmic registration technique, embodied in a programme NEWSUM (Crivellari et al., \$983) allows variable features to be detected against a constant background: 70 Oph $\mathrm{A}$ which shows small variations, must be virtually plage-covered, and in principle allows us to obtain "pure" plage profiles for its spectral type (KOV). In practice one must be careful to eliminate local interstellar medium effects at $\mathrm{H} 3$, which has not been done for 70 Oph $A$.

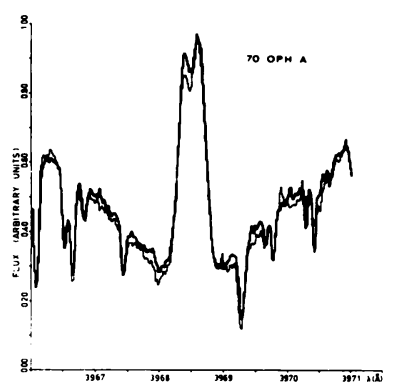

Fig 3: Spectra of 70 Oph $A$ in Ca $H$, separated by 10 nights. This shows strong plage cover and low modulation as plage filling factor is high.

The totally quiescent behaviour of $\alpha$ Cen $A$ points to the final piece in the jigsaw. Its $\mathrm{Ca} \mathrm{H}$ profile, as well as being constant in time, shows similar characteristics to a solar "quiet disc" or integrated solar minimum profile, and we can use it as a reasonable estimate of quiet profiles for stars of type G2V. Our full methodology for filling factor measures is thus: seek out stars with time independent $\mathrm{Ca} \mathrm{H}$ fluxes and "low" profiles. High S:N spectra yield $\Phi_{Q}$. Seek out stars with small variations in fluxes and "high" profiles. High $S: N$ spectra yield $\Phi A$. For stars with flux-varying spectra, monitoring around a period can then give $f P(t)$. Care is needed with asymmetries due to LISM and to the presence of restricted individual plages. References

Wilson, O.C.: 1978, Astrophys. J. 226, 379.

Vaughan, A., Baliuna, S.L., Middelkoop, F., Hartmann, L.W., Mihalas, D., Noyes, R.W., Preston, G.W.: 1981, Astrophys. J. 250, 276.

Crivellari et al.: 1983, Astron. Astrophys. Suppl. Ser. 52, 135. 